\title{
AUTOMAÇÃO DE UM SISTEMA PICK \& PLACE BASEADO EM SISTEMAS A EVENTOS DISCRETOS
}

\author{
Camila R. Ferreira*, Carlos E. V. Nunes ${ }^{\dagger}$ \\ ${ }^{*}$ Rua Prof. Aristides Novis, 2 \\ Federação \\ Salvador, Bahia, Brasil
}

Emails: ribeirocamilaferreira@gmail.com, carlosevnunes@ufba.br

\begin{abstract}
In this paper is realized an automation of a system Pick \& Place using the theory of Discrete Event Systems. The virtual plant, faithful to systems used in industries, it is automated by a real PLC using the conversion from automaton to Ladder Diagram.
\end{abstract}

Keywords- Discrete Event systems, PLC, Ladder Diagram, automata, Automation.

Resumo - Neste artigo é realizado a automação de um sistema Pick $\&$ Place usando a teoria de Sistemas a Eventos Discretos. A planta virtual Pick $\&$ Place, fiel aos sistemas utilizados na indústria, é automatizado por meio de um PLC real utilizando a conversão de autômato em Diagrama Ladder.

Palavras-chave - Sistemas a Eventos Discretos, PLC, Diagrama Ladder, Autômatos, Automação.

\section{Introdução}

Sistemas de manufatura são largamente utilizadas na indústria para executarem atividades repetitivas com precisão, a fim de minimizar custos, prejuízos e acidentes, além de maximizar a qualidade e a produção. Neste contexto, a automação do processo de manufatura permite maior confiabilidade e segurança para a indústria.

A automação de sistemas é realizada observando o comportamento do processo por meio de sensores, de modo que essas informações são enviadas a dispositivos especializados para, posteriormente, elementos conhecidos como atuadores serem acionados no sentido de executar a ação mais correta para o progresso da atividade. Os dispositivos de processamento de sinais mais utilizados na indústria são os PLCs (Programmable Logic Controller)(Music et al., 2005). O PLC é um computador projetado especialmente para o ambiente industrial, pois suporta altas temperaturas, impactos e vibrações, tem imunidade a ruídos elétricos e possui diversas entradas e saídas (SungWook Choi et al., 2008). A dinâmica de funcionamento do PLC é determinado por um programa inserido no dispositivo que pode ser programado de acordo com o padrão IEC 61131 - 3 em cinco diferentes linguagens (ISO/IEC, 2001): Diagrama de Blocos (DB), Diagrama Ladder (LD), Texto estruturado (ST), Lista de Instruções (IL) e Sequential Function Chart (SFC). Entre estas linguagens, o Diagrama Ladder é o mais utilizado na indústria.

Apesar do Diagrama Ladder ser de fácil implementação para sistemas simples, ele se torna mais complicado para sistemas mais complexos, precisando assim de um método sistemático para implementá-lo. Alguns trabalhos mostram a implementação em Ladder utilizando sistemas mo- delados em Sistemas a Eventos Discretos (Liu and Darabi, 2002; S.Peng and Zhou, 2004; Music et al., 2005; Moreira and Basilio, 2014).

Em (Liu and Darabi, 2002) e (?) os autores apresentam um algoritmo para converter modelos em autômato para Diagrama Ladder. Em (S.Peng and Zhou, 2004) e (Moreira and Basilio, 2014) os autores apresentam métodos para transformar modelos em Redes de Petri em Ladder. No entanto, estes trabalhos não apresentam uma implementação em um PLC real.

Neste trabalho, é proposto a automação de um sistema virtual Pick \& Place utilizando a teoria de Sistemas a Eventos Discretos. O modelo do sistema em autômato Moore será convertido em Diagrama Ladder e, posteriormente carregado em um PLC real no sentido de controlar a planta virtual.

Este artigo está organizado como segue: na seção 2 é apresentado os principais conceitos sobre Sistemas a Eventos Discretos (SEDs) e sobre o sistema Pick \& Place, na seção 3 é apresentado os modelos em autômato que compõe o sistema, na seção 4 é apresentado o Diagrama Ladder que controla o sistema, e finalmente na seção 5 são apresentadas as conclusões do trabalho.

\section{Preliminares}

\subsection{Sistemas a Eventos discretos: Definições e notações}

Seja $G=\left\{X, \Sigma, f, \Gamma, x_{0}, X_{m}\right\}$ um autômato que modela um SED. O parâmetro $X$ é o conjunto de estados, $\Sigma$ é o conjunto de eventos, $f: X \times \Sigma \rightarrow X$ é a função de transição, $\Gamma: X \rightarrow 2^{\Sigma}$ é a função de eventos ativos, $x_{0}$ é o estado inicial do sistema e $X_{m}$ é o conjunto de estados marcados (Cassandras and Lafortune, 2008). A linguagem gerada e marcada por $G$ é denotada, respectivamente, por $L$ e 
$L_{m}$.

Suponha uma linguagem $\omega$ definida sob $\Sigma$. Então, o fecho do prefixo de $\omega$ é denotado por $\bar{\omega}$. Se $\omega=\bar{\omega}$, então $\omega$ é dita prefixo fechada. O fecho de Kleene, denotado por $\Sigma^{*}$, é o conjunto de todas as sequências finitas formadas pelos elementos de $\Sigma$, incluindo a sequência fazia $\varepsilon$.

A parte acessível de $G$, denotada por $A c(G)$ é o autômato obtido quando se elimina todos os estados de $G$ que não são alcançados a partir de $x_{0}$, assim como suas transições, ou seja, $A c(G):=$ $\left(X_{a c}, \Sigma, f_{a c}, x_{0}, X_{a c, m}\right)$ em que $X_{a c}=\{x \in X$ : $\left.\left(\exists s \in \Sigma^{*}\right)\left[f\left(x_{0}, s\right)=x\right]\right\}, X_{a c, m}=X_{m} \cap X_{a c} \mathrm{e}$ $f_{a c}=\left.f\right|_{X_{a c} \times \Sigma \rightarrow X_{a c}}$ é a nova função de transição em que o domínio de $f$ é restrito ao domínio dos estados acessíveis. A parte coacessível de $G$, denotado por $\operatorname{CoAc}(G)$, é o autômato obtido quando se elimina todos os estados de $G$ que, a partir destes estados, um estado marcado não é alcançado, ou seja, $\operatorname{CaAc}(G):=\left\{X_{\text {coac }} . \Sigma, f_{\text {coac }}, x_{0, \text { coac }}, X_{m}\right\}$, em que $X_{\text {coac }}=\left\{x \in X:\left(\exists s \in \Sigma^{*}\right)[f(x, s) \in\right.$ $\left.\left.X_{m}\right]\right\}, x_{0, \text { coac }}=x_{0}$ se $x_{0} \in X_{\text {coac }}$ ou $x_{0, \text { coac }}$ é indefinido, caso contrário.

A operação de projeção $P: \Sigma_{g}^{*} \rightarrow \Sigma_{p}^{*}$, em que $\Sigma_{p} \subset \Sigma_{g}$ e a projeção inversa $P^{-1}: \Sigma_{p}^{*} \rightarrow$ $2^{\Sigma_{g}^{*}}$ são definidas de forma usual (Ramadge and Wonham, 1989).

Considere dois autômatos, $G_{1}$ e $G_{2}$. A composição produto e a composição paralela são denotadas por $G_{1} \times G_{2}$ e $G_{1} \| G_{2}$, respectivamente.

Há duas variações de autômatos: autômato Moore e autômato Mealy. No autômato Moore a saída é atribuída a cada estado do autômato, ou seja, as saídas são enviadas quando o autômato entra no estado. No autômato Mealy as saídas são enviadas junto com os eventos de transição do autômato. Na Figura 1 são apresentados os dois tipos de autômatos, note que as saídas são representados por $A$ e $B$.

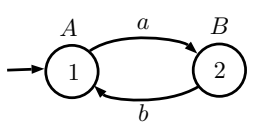

(a)

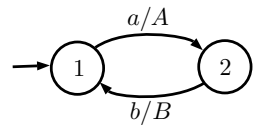

(b)
Figura 1: (a) autômato Moore, (b) autômato Mealy

\subsection{Sistema Pick \& Place: Funcionamento e di- nâmica}

O sistema Pick \& Place, frequentemente utilizado em indústrias de manufatura, são sistemas que tem a função de transferir peças de um lugar para outro sem a intervenção humana. Assim, análises desses sistemas em plataformas virtuais são necessários para evitar danos e para executar uma correta e eficiente implementação.

O ITS PLC (Interactive Training System for $P L C)$ Professional Edition é uma ferramenta virtual utilizada para educação e treino em PLC.
Utilizando gráficos 3D em tempo real e interatividade, esta ferramenta se torna um ambiente virtual muito próxima ao ambiente real. O ITS $P L C$ oferece cinco diferentes plantas industriais, Sorting, Batching, Paletizador, Pick \& Place e Armazem automático, com sensores e atuadores também virtuais para realizar a interação com um PLC real. Esta troca de informações é realizada entre uma placa de aquisição de dados (DAQ) com 32 canais $I / O$ isolados e interface USB (https://realgames.co/).

Para a proposta deste artigo, foi utilizado a planta Pick \& Place do simulador ITS PLC que consiste em duas esteiras paralelas e um manipulador de três eixos, um eixo longitudinal, um eixo transversal e um eixo vertical, como mostrado na Figura 2 (Philippot, 2011). Na esteira (C), esteira de entrada, são transportados três tipos de peças aleatoriamente, peças tipo 1 , tipo 2 e tipo 3 , apresentado na Figura 3. Essas peças são identificadas por um conjunto de dois sensores instalados em (D), enquanto que na esteira (A) é transportado uma caixa quadrada vazia com nove lugares de mesma área, como mostrado na Figura 4, até a esteira (B). O objetivo do sistema é reconhecer o tipo de peça que está sendo transportado na esteira de entrada e transfiri-la para dentro da caixa, que estará parada na esteira (B), de modo que sejam transferidas três peças de cada tipo. As peças devem ser armazenadas na caixa da seguinte maneira: na primeira linha da caixa (L1) devem ser colocadas peças do tipo 1 , na segunda linha da caixa (L2) devem ser colocadas peças do tipo 2 e na terceira linha (L3), peças do tipo 3 .

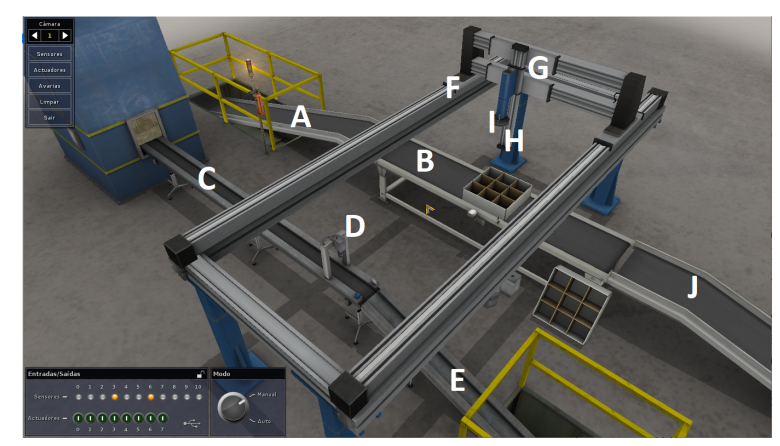

Figura 2: Sistema Pick \& Place

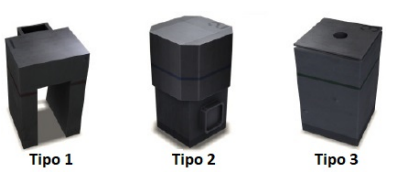

Figura 3: Tipos de peças transportados

Para fazer esta transferência, o manipulador é composto de três eixos: o eixo longitudinal $(\mathrm{F})$, o eixo transversal $(\mathrm{G})$ e o eixo vertical $(\mathrm{H})$. Este último é equipado com um eletroímã (I) que é utilizado para recolher a peça. Caso a primeira linha 


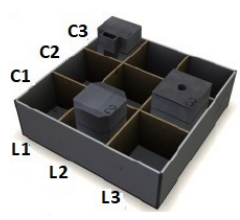

Figura 4: Caixa para alocação de peças na saída

da caixa esteja completa e a esteira (C) transporte uma peça do tipo 1 , então o sistema deve descartá-la pela esteira (E). Da mesma maneira, caso as linhas 2 ou 3 da caixa estejam completas e a esteira (C) transporte peças do tipo 2 ou 3, respectivamente, o sistema deve descartar a peça pela esteira (E). Quando a caixa é completada, a esteira (B) transporta a caixa até a esteira $(\mathrm{J})$, que por sua vez, transporta a caixa com as peças para a saída do sistema.

O sistema Pick \& ${ }^{\prime}$ Place é equipado com nove sensores de presença, $S_{0}$ à $S_{8}$ e oito atuadores com retenção, $A_{0}$ à $A_{7}$. Os sensores $S_{0}$ e $S_{1}$ estão instalados em (D) e atuam na identificação das peças. Se somente $S_{0}$ é ativado, indica passagem da peça do tipo 1 , se somente $S_{1}$ é ativado, indica passagem da peça do tipo 2 e se $S_{0}$ e $S_{1}$ são ativados, indica a passagem da peça do tipo 3 . O sensor $S_{2}$ está colocado no fim da esteira (C) e indica a chegada da peça na posição de coleta pelo manipulador. O sensor $S_{3}$ está alocada na esteira (B) e indica a presença da caixa na zona de preenchimento. O sensor $S_{4}$ está situado no trilho do eixo longitudinal acima da esteira (C) e indica a chegada do manipulador para a coleta da peça. O sensor $S_{5}$ está localizado no eixo transversal e indica a movimentação dos eixos longitudinal ou transversal. O sensor $S_{6}$ está alocado no eixo vertical e detecta o fim do curso superior do eixo indicando que o eletroímã está em cima. O sensor $S_{7}$ também está localizado no eixo vertical e detecta o fim do curso inferior do eixo indicando que o eletroímã está embaixo. O sensor $S_{8}$ está alocado no eletroímã e detecta a presença de peça no dispositivo.

$\mathrm{O}$ atuador $A_{0}$ tem a função de ligar e desligar a esteira $(\mathrm{C})$, o atuador $A_{1}$ liga e desliga as esteiras (A) e (B) ao mesmo tempo, os atuadores $A_{2}$ e $A_{3}$ movimentam o eixo transversal para a direita e para a esquerda, respectivamente. É importante observar que o eixo transversal pode ocupar três posições: a primeira posição, que está mais a esquerda do eixo transversal, a segunda posição, em que o eletroímã fica exatamente no meio do eixo transversal e a terceira posição que se localiza mais a direita do eixo transversal. $\mathrm{O}$ atuador $A_{4}$ movimenta o eixo longitudinal em direção à esteira $(\mathrm{C})$ e o atuador $A_{5}$ movimenta $\mathrm{o}$ eixo longitudinal em direção a esteira (B). Com relação ao eixo logitudinal, o manipulador pode ocupar quatro posições: a primeira posição está localizada acima da esteira (C) e as outras três estão localizadas acima da esteira (B), de modo que a segunda posição fica mais a esquerda da esteira (B), equivalente a posição da coluna ( $\mathrm{C} 1)$ da caixa, a terceira fica no meio da esteira (B), equivalente a posição da coluna $(\mathrm{C} 2)$ da caixa e a quarta fica mais a direita da esteira (B), equivalente a posição da coluna (C3) da caixa. O atuador $A_{6}$ ativa a descida e a subida do eixo vertical e o atuador $A_{7}$ aciona o eletroímã. Assim, a combinação da movimentação dos eixos longitudinal, transversal e vertical permite o armazenamento das peças nos nove espaços da caixa.

O ciclo de funcionamento do sistema Pick \&3 Place é realizado da seguinte maneira: o sistema é iniciado com as esteiras (A), (B) e (C) paradas, o eixo vertical do manipulador se encontra em cima, o eixo transversal está na posição mais a esquerda e o eixo logitudinal está na quarta posição. Assim que o sistema é ligado, as esteiras (A), (B) e (C) entram em funcionamento e o manipulador anda até a esteira (C), pronto para coletar a peça. Durante o transporte das peças pela esteira (C), elas são identificadas em (D) e chegam até o final da esteira sendo detectadas pelo sensor $S_{2}$. A coleta da peça só será executada quando a presença da caixa na zona de preenchimento for detectada pelo sensor $S_{3}$. Assim que a peça é coletada, a esteira (C) se mantém parada e o manipulador anda em direção a esteira (B) e coloca a peça na primeira coluna da caixa (C1), e dependendo da identificação da peça, uma das linhas, L1, L2 ou L3 é escolhida para guardar a peça. Se a peça identificada for do tipo 1 , então a célula da caixa que vai ser preenchida será a célula (L1,C1), se for do tipo 2, então será preenchida a célula (L2,C1) e se for do tipo 3, a célula preenchida será (L3,C1). Depois que o manipulador preenche a célula, ele retorna para coletar outra peça e a esteira (C) volta a funcionar, transportando uma segunda peça. Assim, o manipulador coleta a segunda peça depois que esta for detectada pelo sensor $S_{2}$. Se a segunda peça for do mesmo tipo da primeira, então esta é guardada na coluna $2(\mathrm{C} 2)$ da caixa, caso contrário, será alocado na coluna $1(\mathrm{C} 1)$. Esse ciclo se repete até que as nove células da caixa sejam preenchidas. Se três peças do mesmo tipo já foram guardadas na caixa, mas ela ainda não está completa, então as peças que não precisam ser coletadas são descartadas pela esteira (E). Quando a caixa está completamente preenchida, a esteira (B) volta a funcionar e leva a caixa para ser dispensada pela esteira $(\mathrm{J})$.

\section{Modelo em Sistemas a Eventos Discretos}

Para obter o modelo do sistema Pick \& Place, o sistema foi separado em três módulos menores, módulo da esteira (C), módulo do manipulador e módulo das esteiras (A) e (B). Os modelos desses 
módulos são determinados pela observação comportamental do processo e são denotados por $E_{1}$, $M$ e $E_{2}$, respectivamente, e o modelo completo do sistema é obtido pela operação paralelo entre os três módulos, no entanto, como o modelo completo possui 67 estados, ele não será apresentado neste trabalho devido a limitações de espaço.

Para auxiliar na construção do modelo em autômato do sistema, foram usados seis contadores, $C_{0}, C_{1}, C_{2}, C_{3}, C_{4}$ e $C_{5}$ disponíveis do Diagrama Ladder, associados aos eventos $c_{0}, c_{1}, c_{2}$, $c_{3}, c_{4}$ e $c_{5}$. Esses contadores são utilizados para contar quantas peças foram guardadas na caixa de modo que, para extrair esta informação, os contadores são utilizados em pares. Para as peças do tipo 1, são utilizados os contadores $C_{0}$ e $C_{3}$, para as peças do tipo 2 , os contadores $C_{1}$ e $C_{4}$ e para peças do tipo 3 , os contadores $C_{3}$ e $C_{5}$. Se o sistema guardou uma peça do tipo 1 , os contadores $C_{0}$ e $C_{3}$ terão saídas booleanas iguais a zero, de modo que os eventos associados serão representados por $\overline{c_{0}}$ e $\overline{c_{3}}$, se for guardada duas peças do tipo um, a saída booleada de $C_{0}$ será zero e de $C_{3}$ será um, assim os eventos serão representados por $\overline{c_{0}}$ e $c_{3}$ e se for três peças, as saídas de $C_{0}$ e $C_{3}$ terão saídas iguais a um e são representados pelos eventos $c_{0}$ e $c_{3}$. O modelo da esteira (C) é mostrado na Figura 5.

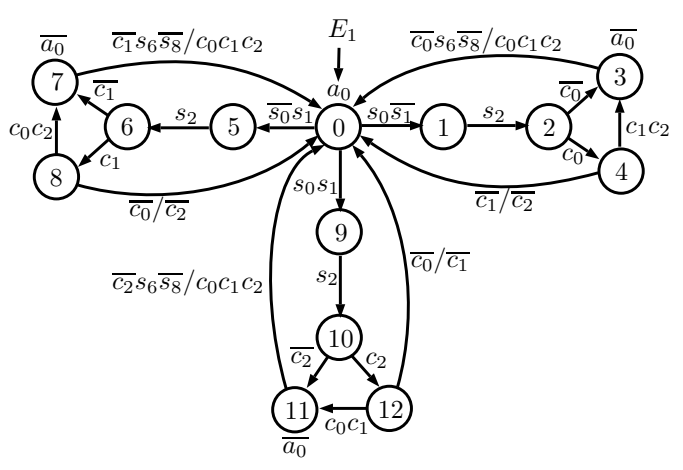

Figura 5: Modelo da esteira (C)

Neste modelo os eventos compostos por $s_{i}$, $i=0,1,2, \ldots, 8$, são eventos que representam a leitura dos sensores $S_{i}$, explicados na seção 2 , de maneira que, o evento $s_{i}$ indica que o sensor $S_{i}$ identificou o objeto e $\overline{s_{i}}$ indica que não há objeto a ser identificado pelo sensor $S_{i}$. Os eventos formados pela leitura de mais de um sensor, como o evento $\left\{\overline{c_{1}} s_{6} \overline{s_{8}}\right\} \in \Gamma(7)$, indica que a transição só ocorre se as três leituras forem verdadeiras, ou seja, se o sensor $S_{6}$ identifica presença, se o sensor $S_{8}$ não identifica presença e se o contador $C_{1}$ não identificar a quantidade de peças guardadas.

Os contadores foram utilizados como sensor virtual para identificar quantas peças foram coletadas pelo manipulador e, consequentemente, guardadas na caixa. Os eventos formados por mais de um contador como o evento $\overline{c_{0}} / \overline{c_{1}} \in \Gamma(12)$ indica que a transição ocorre se $\overline{c_{0}}$ ou $\overline{c_{1}}$ é verdadeiro. A saída do autômato é executada nos estados $0,3,7$ e 11 ativando ou desativando o atuador $A_{0}$ representado pelo evento $a_{0}$, que indica que a esteira (C) é ligada, e o evento $\overline{a_{0}}$ que indica que a esteira (C) é desligada.

Os estados do autômato $E_{1}$ representam o seguinte: o estado inicial, 0 , indica que a esteira (C) está transportando peças aleatórias, o estado 1 indica que a peça do tipo 1 foi identificada, o estado 2 aponta que a peça do tipo 1 chegou na posição de coleta do manipulador, o estado 3 assinala que a esteira está esperando o manipulador coletar a peça e no estado 4 o sistema verifica se as peças do tipo 2 ou 3 já foram coletadas três vezes. Os estados 5, 6, 7 e 8 tem o mesmo significado dos estados $1,2,3$ e 4, porém com relação a peças do tipo 2 e os estados 9, 10, 11 e 12 com relação às peças do tipo 3 .

O modelo do manipulador contem 60 estados e não será possível apresenta-lo por completo neste artigo por falta de espaço. No entanto, é apresentado na Figura 6 a parte do autômato referente ao transporte de peças do tipo 3 da esteira $(\mathrm{C})$ para a caixa na esteira (B). As partes do autômato ocultadas são referentes às peças do tipo 1 e 2 e são semelhantes à parte apresentada.

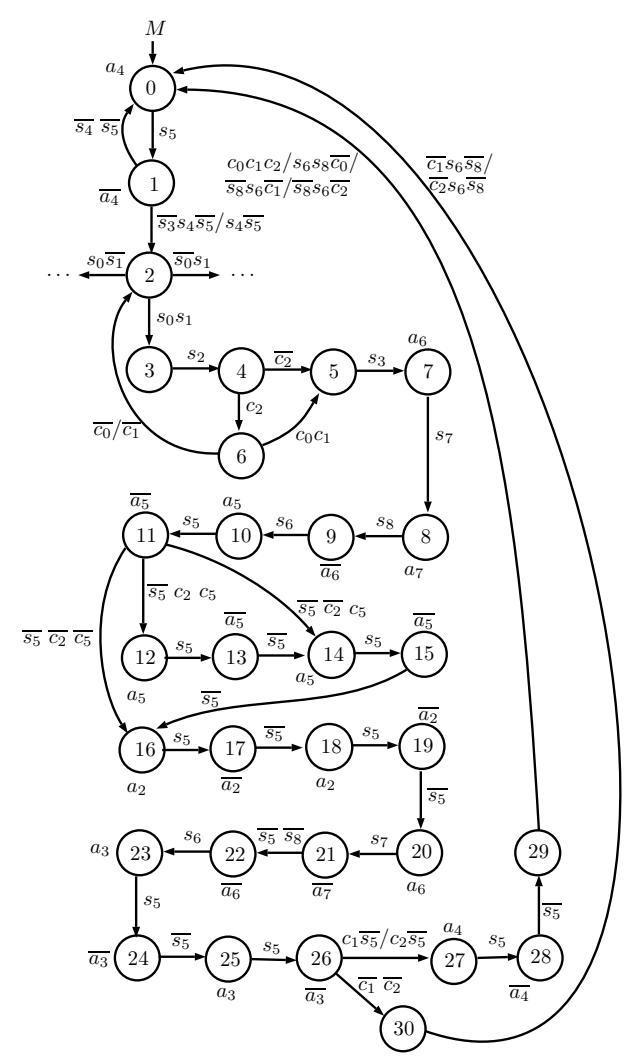

Figura 6: Modelo do manipulador

Os estados do autômato $M$ representam o seguinte: o estados 0 e 1 inicia o sistema levando o manipulador para a esteira (C), no estado 2 as peças são identificadas. Nos estados 3,4,5 e 6 , o 
autômato identifica a peça do tipo 3 e verifica se já pegou três peças deste tipo, no estado 7,8 e 9 a peça é coletada, nos estados 10 e 11, o manipulador vai para a esteira (B) com a peça, nos estados 12, 13, 14 e 15, o autômato seleciona qual coluna da caixa a peça será guardada, nos estados 16, 17, 18 e 19, o manipulador vai até a linha três da caixa (L3), nos estados 20, 21 e 22, o mainipulador guarda a peça na caixa, nos estados 23, 24, 25 e 26 , o manipulador retorna para a posição da linha um (L1) da caixa e verifica se já pegou três peças do tipo 2 e 3 e, finalmente, nos estados 27 , 28, 29 e 30, o manipulador retorna para a esteira (C) para pegar outra peça.

$\mathrm{O}$ autômato $E_{2}$ é mostrado na Figura 7 e seus estados representam o seguinte: no estado 0 inicia o sistema ligando a esteira (A) e (B), no estado 1 o autômato espera a caixa chegar na zona de preenchimento, no estado 2 a caixa chega na zona de preenchimento e a esteira desliga, no estado 3 o autômato verifica se a caixa está completa ou não e no estado 4 o autômato sabe que a caixa está incompleta e retorna para o estado 1.

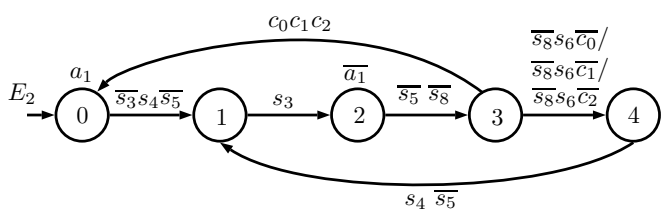

Figura 7: Modelo da esteira (A) e (B)

\section{Diagrama Ladder}

O diagrama Ladder é definida na norma IEC 61131-3 e entre as diversas instruções fornecidas por esta linguagem, foi utilizado para este trabalho instruções como contatores normalmente aberto (NA), contatores normalmente fechado (NF), bobinas e contadores. Os contadores foram utilizadas para auxiliar na contagem de peças colocadas na caixa e possuem dois parâmetros: preset e acumulador. O preset deve ser configurado pelo programador e indica o limite de contagens que o contador irá executar e o acumulador mostra quantas contagens o contador já fez. Toda vez que a lógica da linha for verdadeira, a bobina de saída do contador, CU, se torna 1, caso contrário, se mantém em 0 e o acumulador incrementa uma unidade. Quando o acumulador alcança o valor de preset, outra saída do contador, DN, se torna 1 e esta se mantem até que alguma outra lógica reinicie o acumulador por uma bobina de reset.

A conversão do modelo em autômato para o Diagrama Ladder é feita de maneira direta e, na literatura, pode-se encontrar alguns métodos bem estabelecidos. Neste trabalho, será usado um método baseado em (Uzam et al., 2009) para converter autômato Moore em Diagrama Ladder. Nesta metodologia, variáveis são atribuídas aos estados e às transições do autômato e está exemplificada na Figura 8.

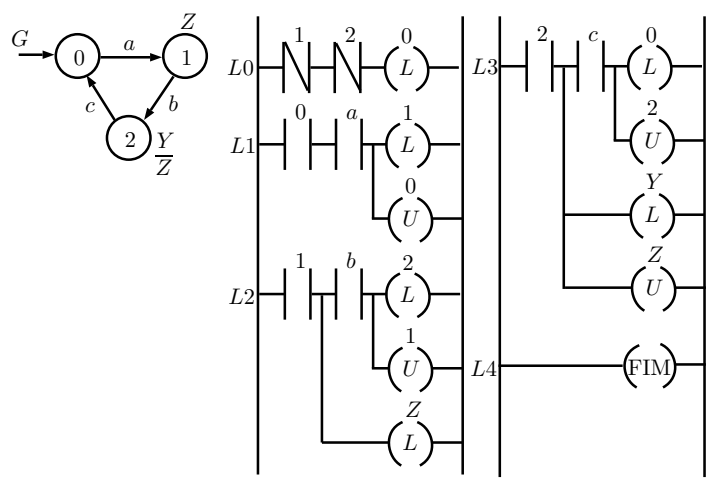

Figura 8: Conversão de autômato para Diagrama Ladder

O Diagrama Ladder usado para controlar o sistema Pick \& Place foi gerado a partir do modelo completo do sistema em autômato e foi carregado no PLC Allen Bradley da série Micrologix 1000 e é mostrado na Figura 9. Nesta figura, nas linhas $L 0$ a $L 2$ o sistema é iniciado ligando as esteira (A), (B) e (C) e deslocando o manipulador para a esteira (C), na linha $L 3$ as peças são identificadas.

Supõe-se que a primeira peça a ser identificada seja a peça do tipo 1 . Na linha $L 4$ a peça chega no final da esteira (B) e é detectada pelo sensor $S_{2}$, nas linhas $L 5$ e $L 6$ é verificado se já existem três peças dos tipos 1,2 e 3 na caixa, se tiver, os contadores são resetados, na linha $L 7$ a caixa é identificada na zona de preenchimento, nas linhas $L 8$ a $L 10$ o manipulador pega a peça na esteira (C) e os contadores $C_{0}$ e $C_{3}$ incrementam a contagem em uma unidade, na linha $L 11$ o manipulador retorna para a esteira (B) ocupando a posição acima da célula $(\mathrm{L} 1, \mathrm{C} 1)$, na linha $L 12$ o sistema verifica se já pegou uma, duas ou três peças do tipo 1. Se for a primeira peça, o Ladder vai para a linha $L 13$, se for a segunda peça, o Ladder vai para a linha $L 19$ e se for a terceira peça, o $L a d d e r$ vai para a linha $L 21$. Nas linhas $L 13, L 14$ e $L 15$, o manipulador deixa a peça na caixa, na célula (L1,C1), e retorna para a esteira (C), além de verificar se já existem três peças do tipo 1 na caixa. Se existir três peças do tipo 1 na caixa, o contador $C_{3}$ é resetado e o Ladder segue para a linha $L 16$, caso contrário segue para as linhas $L 66$ e $L 67$. As linhas $L 66$ e $L 77$ são equivalentes às linhas $L 1$ e $L 2$, porém mantendo as esteiras (A) e (B) paradas. Então o Ladder retorna à linha L3 e espera a segunda peça transportada ser identificada. Nas linhas $L 16$ e $L 17$ é verificado se já tem três peças do tipo 2 e do tipo 3, então o Ladder retorna para a linha $L 1$ ou $L 3$.

Supondo que a segunda peça a ser transportada também é do tipo 1, o Ladder percorre as linhas $L 4$ até $L 11$ como já explicado anteriormente. $\mathrm{Na}$ linha $L 12$, é verificado que esta é a segunda 
peça e o Ladder segue para as linhas L19 e L20 que faz o manipulador ir para a célula (L1,C2), para guardar a peça nesta célula o Ladder retorna às linhas L13 à L15. Então o Ladder reinicia o ciclo na linha $L 3$. Se a terceira peça for do tipo 1 , o Ladder percorre as linhas L4 à L11 e na linha $L 12$ é verificado que é a terceira peça, então segue para as linhas $L 21, L 22, L 19$ e $L 20$ que leva o manipulador para a célula (L1,C3), então se repete as linhas $L 13$ até $L 15$. Na linha $L 15$ é verificado que a caixa já tem três peças do tipo 1 e o contador $C_{3}$ é resetado, na linha $L 16$ é verificado se já tem três peças do tipo 2 e na linha $L 17$ é verificado se já exitem três peças do tipo 3 na caixa, assim, na linha $L 18$ é verificado se a caixa já está cheia. Se tiver cheia, o Ladder retorna para a linha $L 0$, caso contrário retorna para a linha $L 65$ e então para a linha $L 3$.

Se uma peça do tipo 2 for transportada pela esteira (C), ela será identificada na linha $L 3$ e o Ladder segue para a linha $L 23$. As linhas $L 23$ à L31 são equivalentes as linhas $L 4$ a $L 12$. Se for a primeira peça do tipo 2 o Ladder segue para as linhas L32 até L36, que faz o manipulador guardar a peça na célula (L2,C1), na linha L37 o eixo transversal retorna para a primeira posição do eixo (mais a esquerda) e nas linhas $L 38, L 39, L 16, L 17$ e $L 18$ é verificado se a caixa está cheia. Se estiver cheia, o ciclo recomeça na linha $L 1$, caso contrário o ciclo recomeça na linha $L 3$. Supondo a segunda peça do tipo 2, o Ladder segue o mesmo caminho anterior, porém da linha L31 segue para as linhas $L 40, L 41, L 32$ até $L 35$ para guardar a peça na célula $(\mathrm{L} 2, \mathrm{C} 2)$ e retorna para o início do ciclo como explicado anteriormente. Para a terceira peça do tipo 2, o Ladder segue o mesmo caminho das peças anteriores, porém ela é guardada na célula (L2,C3) utilizando as linhas L42, L43, L40 e L41 para alcançar a célula (L2,C3) e as linhas L32 à L36 para guardá-la.

Para as peças do tipo 3, o Ladder utiliza as linhas a partir de $L 44$. As linhas $L 44$ à $L 52$ é semelhante às linhas $L 4$ à $L 12$. Para a primeira peça do tipo 3, o Ladder segue para as linhas $L 53$ até $L 56$ e alcança a célula $(\mathrm{L} 3, \mathrm{C} 1)$ e nas linhas $L 57$ até $L 59$ a peça é guardada. Nas linhas $L 60$, $L 61, L 37$ e $L 38$, o eixo transversal retorna para a primeira posição do eixo. Para a segunda peça do tipo 3, nas linhas L62 e L63 o manipulador alcança a célula (L3,C2) e a peça é guardada como explicado anteriormente. Para a terceira peça do tipo 3, o Ladder utiliza as linhas L64, L65, L62 e L63 para o manipulador chegar na célula (L3,C3). Então o ciclo recomeça na linha $L 1$.

\section{Conclusões e trabalhos futuros}

Neste trabalho foi realizado a automação de um sistema Pick \& Place usando teoria de Sistemas a Eventos discretos. A automação foi realizado por um PLC real que, usando Diagrama Ladder, con- trola o sistema em uma plataforma virtual. Assim, com este sistema controlado, é possível realizar outros estudos e ensaios relacionados ao tema de Sistemas a Eventos Discretos como diagnóstico de falhas, controle supervisório ou análise de atrasos de comunicação no sentido de realizar comparações para avaliar características de performance.

\section{Referências}

Cassandras, C. G. and Lafortune, S. (2008). Introduction to Discrete Event Systems, $2^{\circ}$ edn, Springer.

ISO/IEC (2001). Programmable logic controllers, IEC 61131-3.

Liu, J. and Darabi, H. (2002). Ladder logic implementation of ramadge-wonham supervisory controller, Sixth International Workshop on Discrete Event Systems, 2002. Proceedings., pp. 383-389.

Moreira, M. V. and Basilio, J. C. (2014). Bridging the gap between design and implementation of discrete-event controllers, IEEE Transactions on Automation Science and Engineering 11(1): 48-65.

Music, G., Gradisar, D. and Matko, D. (2005). Iec 61131-3 compliant control code generation from discrete event models, Proceedings of the 2005 IEEE International Symposium on, Mediterrean Conference on Control and Automation Intelligent Control, 2005., pp. 346351.

Philippot, A. (2011). Survey on diagnosis of a pick and place benchmark: Special session on diagnosis of discrete event systems: Application on a benchmark, 2011 3rd International Workshop on Dependable Control of Discrete Systems, pp. 25-28.

Ramadge, P. J. G. and Wonham, W. M. (1989). The control of discrete event systems, Proceedings of the IEEE 77(1): 81-98.

S.Peng, S. and Zhou, M. Z. (2004). Ladder diagram and petri-net-based discrete-event control design methods, IEEE Transactions on Systems, Man, and Cybernetics, Part C (Applications and Reviews) 34(4): 523-531.

Sung-Wook Choi, Kang-Gu Lee, Lock-Jo Koo, Chang-Mok Park, Park, S. and Gi-Nam Wang (2008). Ladder diagram generation using generic model of discrete event system, 2008 rth IEEE International Conference on Cybernetic Intelligent Systems, pp. 1-4.

Uzam, M., Gelen, G. and Dalci, R. (2009). Timed transition automata and their ladder logic implementation, 2009 XXII International Symposium on Information, Communication and Automation Technologies, pp. 1-8. 


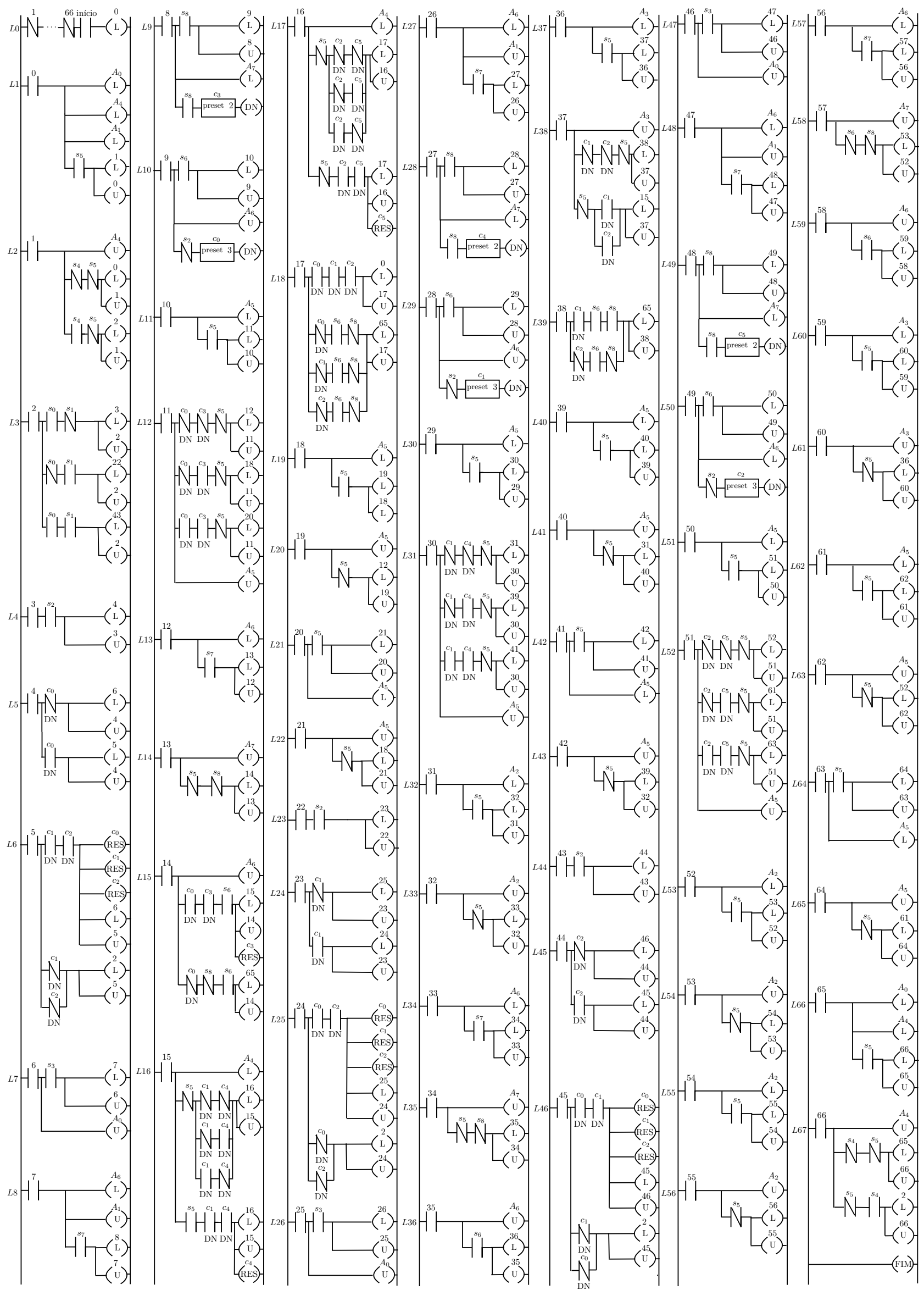

Figura 9: Diagrama Ladder para controle do sistema Pick $\&$ place 